\title{
EXTREMES OF ORDER STATISTICS OF STATIONARY GAUSSIAN PROCESSES*
}

BY

\author{
CHUNMING Z HAO (ChENGdu)
}

Abstract. Let $\left\{X_{i}(t), t \geqslant 0\right\}, 1 \leqslant i \leqslant n$, be mutually independent and identically distributed centered stationary Gaussian processes. Under some mild assumptions on the covariance function, we derive an asymptotic expansion of

$$
\mathbb{P}\left(\sup _{t \in\left[0, x m_{r}(u)\right]} X_{(r)}(t) \leqslant u\right) \quad \text { as } u \rightarrow \infty,
$$

where

$$
m_{r}(u)=\left(\mathbb{P}\left(\sup _{t \in[0,1]} X_{(r)}(t)>u\right)\right)^{-1}(1+o(1)),
$$

and $\left\{X_{(r)}(t), t \geqslant 0\right\}$ is the $r$ th order statistic process of $\left\{X_{i}(t), t \geqslant 0\right\}$, $1 \leqslant i, r \leqslant n$. As an application of the derived result, we analyze the asymptotics of supremum of the order statistic process of stationary Gaussian processes over random intervals.

2010 AMS Mathematics Subject Classification: Primary: 60G15; Secondary: 60G70.

Key words and phrases: Asymptotic, Gaussian processes, order statistic, stationarity, supremum.

\section{INTRODUCTION}

Let $\{X(t): t \geqslant 0\}$ be a centered stationary Gaussian process with continuous sample paths. One of the classical results in extreme value theory states that, under some mild conditions on the covariance function of $X$,

$$
\lim _{u \rightarrow \infty} \mathbb{P}\left(\sup _{t \in[0, x m(u)]} X(t) \leqslant u\right)=e^{-x}
$$

for $x>0$ and $m(u)=\mathbb{P}\left(\sup _{t \in[0,1]} X(t)>u\right)^{-1}$; see, e.g., Leadbetter et al. [II], Theorem 12.3.4; Arendarczyk and Dębicki [4], Lemma 4.3; Tan and Hashorva [13], Lemma 3.3.

\footnotetext{
* This work was supported by the FP7 project RARE-318984.
} 
Consider a vector-valued Gaussian stochastic process $\{\mathbf{X}(t): t \geqslant 0\}$, where $\mathbf{X}(t)=\left(X_{1}(t), \ldots, X_{n}(t)\right)$ with $\left\{X_{i}(t): t \geqslant 0\right\}, i=1, \ldots, n$, being mutually independent copies of $\{X(t): t \geqslant 0\}$. Denote by $\left\{X_{(r)}(t), t \geqslant 0\right\}, r=1,2, \ldots, n$, the $r$ th smallest order statistic process, i.e., for each $t \geqslant 0$,

$$
X_{(1)}(t)=\min _{1 \leqslant i \leqslant n} X_{i}(t) \leqslant X_{(2)}(t) \leqslant \ldots \leqslant \max _{1 \leqslant i \leqslant n} X_{i}(t)=X_{(n)}(t) .
$$

In this contribution we derive a counterpart of (ILI) for $\left\{X_{(r)}(t), t \geqslant 0\right\}$.

One of important motivations to analyze asymptotic properties of extremes of order statistic processes is their relation with the conjunction problem. Following [14], the set of conjunctions $C_{T, u}$ is defined as

$$
C_{T, u}:=\left\{t \in[0, T]: \min _{1 \leqslant i \leqslant n} X_{i}(t)>u\right\},
$$

so

$$
\mathbb{P}\left(C_{T, u}=\emptyset\right)=\mathbb{P}\left(\sup _{t \in[0, T]} \min _{1 \leqslant i \leqslant n} X_{i}(t) \leqslant u\right) .
$$

We refer to [2], [3], [6], [9], [14] for recent results on asymptotic properties of $\mathbb{P}\left(C_{T, u} \neq \emptyset\right)$.

As an application of the obtained result we provide the exact asymptotics of

$$
\mathbb{P}\left(\sup _{t \in[0, \mathcal{T}]} X_{(r)}(t)>u\right) \quad \text { as } u \rightarrow \infty
$$

for $\mathcal{T}$ being a nonnegative random variable independent of $\mathbf{X}(t)$. The obtained asymptotics extends the recent results of Arendarczyk and Dębicki [4].

\section{PRELIMINARIES}

Suppose that $\mathbf{X}(t)=\left(X_{1}(t), \ldots, X_{n}(t)\right)$ and $\left\{X_{i}(t): t \geqslant 0\right\}, i=1, \ldots, n$, are mutually independent centered stationary Gaussian processes with covariance function $r(t)$ satisfying the following conditions:

(A1) $r(t)=1-t^{\alpha}+o\left(t^{\alpha}\right)$ as $t \rightarrow 0$;

(A2) $r(t)<1$ if $t>0$;

(A3) $r(t) \log t \rightarrow 0$ as $t \rightarrow \infty$.

Following Dębicki et al. [9], let us introduce the generalized Pickands constant as

where

$$
\mathcal{H}_{\alpha, k}=\lim _{S \rightarrow \infty} S^{-1} \mathcal{H}_{\alpha, k}(S) \in(0, \infty),
$$

$$
\begin{aligned}
& \mathcal{H}_{\alpha, k}(S) \\
= & \int_{R^{n}} \exp \left(\sum_{i=1}^{k} w_{j}\right) \mathbb{P}\left(\sup _{t \in[0, S]} \min _{1 \leqslant i \leqslant k}\left(\sqrt{2} B_{\alpha}^{(i)}(t)-t^{\alpha}-w_{i}\right)>0\right) d \mathbf{w} \in(0, \infty),
\end{aligned}
$$


and $B_{\alpha}^{(i)}, i=1, \ldots, n$, are mutually independent standard fractional Brownian motions with Hurst index $\alpha / 2 \in(0,1]$, i.e., centered Gaussian processes with stationary increments and variance function $t^{\alpha}$.

Let

$$
m_{r}(u):=\frac{(2 \pi)^{(n+1-r) / 2}}{c_{n, r-1} \mathcal{H}_{\alpha, n+1-r}} u^{n+1-r-2 / \alpha} \exp \left(\frac{n+1-r}{2} u^{2}\right),
$$

where

$$
c_{n, r-1}=\frac{n !}{(r-1) !(n+1-r) !} .
$$

It follows from Theorem 2.2 in [ 8$]$ that, for each $T>0$ and $1 \leqslant r \leqslant n$,

$$
\begin{aligned}
\mathbb{P}\left(\sup _{t \in[0, T]} X_{(r)}(t)>u\right) & =c_{n, r-1} \mathcal{H}_{\alpha, n+1-r} T u^{2 / \alpha}(\Psi(u))^{n+1-r}(1+o(1)) \\
& =\frac{T}{m_{r}(u)}(1+o(1)) \quad \text { as } u \rightarrow \infty
\end{aligned}
$$

where $\Psi(u)=\frac{1}{\sqrt{2 \pi}} \int_{u}^{\infty} \exp \left(-x^{2} / 2\right) d x$.

\section{MAIN RESULTS}

The following theorem constitutes the main result of this contribution.

THEOREM 3.1. Let $\left\{X_{j}(t), t \geqslant 0\right\}$ be independent and identically distributed centered stationary Gaussian processes with convariance function $r(t)$ satisfying the conditions (A1)-(A3) and assume that $0<A<B<\infty$ and $x>0$. Then

$$
\mathbb{P}\left(\sup _{t \in\left[0, x m_{r}(u)\right]} X_{(r)}(t) \leqslant u\right) \rightarrow e^{-x} \quad \text { as } u \rightarrow \infty,
$$

uniformly for $x \in[A, B]$.

Let $\mathcal{T}$ be a nonnegative random variable which is independent of $\mathbf{X}$. In the following theorem we discuss the asymptotic behavior of $\mathbb{P}\left(\sup _{t \in[0, \mathcal{T}]} X_{(r)}(t)>u\right)$ as $u \rightarrow \infty$. It appears that the qualitative form of the asymptotics strongly depends on heaviness of the tail of $\mathcal{T}$.

THEOREM 3.2. Let $\left\{X_{j}(t), t \geqslant 0\right\}$ be independent and identically distributed centered stationary Gaussian processes with convariance function $r(t)$ satisfying the conditions (A1)-(A3), and let $\mathcal{T}$ be a nonnegative random variable independent of $X$.

(i) If $\mathbb{E} \mathcal{T}<\infty$, then, as $u \rightarrow \infty$,

$$
\mathbb{P}\left(\sup _{t \in[0, \mathcal{T}]} X_{(r)}(t)>u\right)=\mathbb{E} \mathcal{T} c_{n, r-1} \mathcal{H}_{\alpha, n+1-r} u^{2 / \alpha}(\Psi(u))^{n+1-r}(1+o(1)) .
$$


(ii) If $\mathcal{T}$ has a regularly varying tail distribution at infinity with index $\lambda \in$ $(0,1)$, then, as $u \rightarrow \infty$,

$$
\mathbb{P}\left(\sup _{t \in[0, \mathcal{T}]} X_{(r)}(t)>u\right)=\Gamma(1-\lambda) \mathbb{P}\left(\mathcal{T}>m_{r}(u)\right)(1+o(1)) .
$$

(iii) If $\mathcal{T}$ has a slowly varying tail distribution at infinity, then, as $u \rightarrow \infty$,

$$
\mathbb{P}\left(\sup _{t \in[0, \mathcal{T}]} X_{(r)}(t)>u\right)=\mathbb{P}\left(\mathcal{T}>m_{r}(u)\right)(1+o(1)) .
$$

The proofs of Theorems 3.1 and 3.2 are given in Section 4 .

\section{PROOFS}

Before proceeding to the proofs of Theorems 3.1$]$ and B.2, we give some preliminary lemmas. Let us put $\mathcal{T}_{r}=x m_{r}(u)$ and $n_{r}=\left\lfloor\mathcal{T}_{r}\right\rfloor$. For any $\varepsilon \in(0,1)$ and $1 \leqslant l \leqslant n_{r}$, we write $I_{l}=[l-1+\varepsilon, l]$ and $I_{l}^{*}=[l-1, l-1+\varepsilon]$.

Lemma 4.1. For each $B>A>0$,

$$
\lim _{u \rightarrow \infty}\left|\mathbb{P}\left(\sup _{t \in\left[0, n_{r}\right]} X_{(r)}(t) \leqslant u\right)-\mathbb{P}\left(\sup _{t \in \bigcup_{l=1}^{n_{r}} I_{l}} X_{(r)}(t) \leqslant u\right)\right| \leqslant \rho_{1}(\varepsilon),
$$

uniformly for $x \in[A, B]$, where $\rho_{1}(\varepsilon) \rightarrow 0$ as $\varepsilon \rightarrow 0$.

Pr o of. Suppose that $x \in[A, B]$. By stationarity, Bonferroni's inequality (see, e.g., [10]) and (2.2), we have

$$
\begin{aligned}
0 & \leqslant \mathbb{P}\left(\sup _{t \in \bigcup_{l=1}^{n_{r}} I_{l}} X_{(r)}(t) \leqslant u\right)-\mathbb{P}\left(\sup _{t \in\left[0, n_{r}\right]} X_{(r)}(t) \leqslant u\right) \\
& =\mathbb{P}\left(\sup _{t \in\left[0, n_{r}\right]} X_{(r)}(t)>u\right)-\mathbb{P}\left(\sup _{t \in \bigcup_{l=1}^{n_{r}} I_{l}} X_{(r)}(t)>u\right) \\
& \leqslant \mathbb{P}\left(\sup _{t \in \bigcup_{l=1}^{n_{r}} I_{l}^{*}} X_{(r)}(t)>u\right) \leqslant n_{r} \mathbb{P}\left(\sup _{t \in[0, \varepsilon]} X_{(r)}(t)>u\right) \\
& =x m_{r}(u) \frac{\varepsilon}{m_{r}(u)}(1+o(1)) \leqslant B \varepsilon=: \rho_{1}(\varepsilon) \quad \text { as } u \rightarrow \infty .
\end{aligned}
$$

This completes the proof.

LEMMA 4.2. Let $q=q(u)=a u^{-2 / \alpha}$ for some $a>0$. Then

$$
\limsup _{u \rightarrow \infty}\left|\mathbb{P}\left(\sup _{t \in \bigcup_{l=1}^{n_{r}} I_{l}} X_{(r)}(t) \leqslant u\right)-\mathbb{P}\left(\max _{i q \in \bigcup_{l=1}^{n_{r}} I_{l}} X_{(r)}(i q) \leqslant u\right)\right| \leqslant \rho_{2}(a),
$$

uniformly for $x \in[A, B]$, where $\rho_{2}(a) \rightarrow 0$ as $a \rightarrow 0$. 


$$
\begin{aligned}
& \text { P r o o f. Since } X_{i}(t) \text { are independent and identically distributed, we obtain } \\
& \mathbb{P}\left(\max _{i q \in I_{1}} X_{(r)}(i q)>u\right) \\
= & \mathbb{P}\left(\bigcup_{i q \in I_{1}} \bigcup_{j=n-r+1}^{n}\left\{\exists k_{1}, \ldots, k_{j}, X_{k_{1}}(i q)>u, \ldots, X_{k_{j}}(i q)>u\right\}\right) \\
= & \mathbb{P}\left(\bigcup _ { i q \in I _ { 1 } } \bigcup _ { j = n - r + 1 } ^ { n } \left\{\exists k_{1}, \ldots, k_{j}, X_{k_{1}}(i q)>u, \ldots, X_{k_{j}}(i q)>u,\right.\right. \\
= & \left.\left.\sum_{j=}^{n-r+1} X_{k}(i q) \leqslant u, k \neq k_{1}, \ldots, k_{j}\right\}\right) \\
= & \sum_{j=}^{n} c_{n-r+1}^{n} \mathbb{P}\left(\exists_{i q \in I_{1}}, X_{1}(i q)>u, \ldots, X_{j}(i q)>u, X_{k}(i q) \leqslant u, k>j\right)
\end{aligned}
$$

Following Dębicki et al. [8] we define

$$
\mathcal{H}^{\prime} \alpha, j(a)=\frac{1}{a} P\left(\max _{k \geqslant 1} \min _{1 \leqslant m \leqslant j}\left(\sqrt{2} B_{\alpha}^{(m)}(a k)-(a k)^{\alpha}+\eta_{m}\right) \leqslant 0\right),
$$

where $j=1,2, \ldots, n$, and $\left\{B_{\alpha}^{(m)}, t \geqslant 0\right\}, m \geqslant 1$, are independent and identically distributed standard fractional Brownian motions which are further independent of independent unit exponential random variables $\eta_{m}$. Using analogous arguments to those in the proof of Theorem 1.1 in Dębicki et al. [8] or Lemma 1 in Albin and Choi [1], we have

$$
\begin{aligned}
\mathbb{P}\left(\max _{i q \in I_{1}} X_{(r)}(i q)>u\right) & =\sum_{j=n-r+1}^{n} \frac{\mathcal{H}_{\alpha, j}^{\prime}(a)}{\mathcal{H}_{\alpha, j}} \frac{1-\varepsilon}{m_{n+1-j}(u)} \\
& =\frac{\mathcal{H}^{\prime}{ }_{\alpha, n+1-r}(a)}{\mathcal{H}_{\alpha, n+1-r}} \frac{1-\varepsilon}{m_{r}(u)}(1+o(1)) \quad \text { as } u \rightarrow \infty,
\end{aligned}
$$

where $\mathcal{H}^{\prime}{ }_{\alpha, k}(a) \rightarrow \mathcal{H}_{\alpha, k}$ as $a \rightarrow 0$. Therefore, by stationarity, we obtain

$$
\begin{aligned}
0 \leqslant & \mathbb{P}\left(\max _{i q \in \bigcup_{l=1}^{n_{r}} I_{l}} X_{(r)}(i q) \leqslant u\right)-\mathbb{P}\left(\sup _{t \in \bigcup_{l=1}^{n_{r}} I_{l}} X_{(r)}(t) \leqslant u\right) \\
\leqslant & n_{r} \max _{1 \leqslant l \leqslant n_{r}}\left(\mathbb{P}\left(\max _{i q \in I_{l}} X_{(r)}(i q) \leqslant u\right)-\mathbb{P}\left(\sup _{t \in I_{l}} X_{(r)}(t) \leqslant u\right)\right) \\
\leqslant & n_{r} \mathbb{P}\left(X_{(r)}(0)>u\right)+n_{r} \mathbb{P}\left(\sup _{t \in[0,1-\varepsilon]} X_{(r)}(t)>u\right) \\
& -n_{r} \mathbb{P}\left(\max _{i q \in[0,1-\varepsilon]} X_{(r)}(i q)>u\right) \\
= & x m_{r}(u)\left(o\left(\frac{1}{m_{r}(u)}\right)+\frac{1-\varepsilon}{m_{r}(u)}-\frac{\mathcal{H}^{\prime}{ }_{\alpha, n+1-r}(a)}{\mathcal{H}_{\alpha, n+1-r}} \frac{1-\varepsilon}{m_{r}(u)}\right)(1+o(1)) \\
\leqslant & B\left(1-\frac{\mathcal{H}_{\alpha, n+1-r}^{\prime}(a)}{\mathcal{H}_{\alpha, n+1-r}}\right)=: \rho_{2}(a),
\end{aligned}
$$


where the penultimate expression is due to (2.2). Since $\rho_{2}(a) \rightarrow 0$ as $a \rightarrow 0$, the proof is completed.

For each $1 \leqslant j \leqslant n$, let $\left\{X_{j}^{(k)}(t), t \geqslant 0\right\}_{k=1}^{\infty}$ be a sequence of independent and identically distributed centered stationary Gaussian processes that satisfy the conditions (A1)-(A3). Define

$$
Y_{j}(t)=X_{j}^{(k)}(t) \quad \text { if } t \in[k-1, k),
$$

and, for $t \geqslant 0$,

$$
Y_{(1)}(t)=\min _{1 \leqslant j \leqslant n} Y_{j}(t) \leqslant Y_{(2)}(t) \leqslant \ldots \leqslant \max _{1 \leqslant j \leqslant n} Y_{j}(t)=Y_{(n)}(t) .
$$

\section{LEMMA 4.3. We have}

$$
\lim _{u \rightarrow \infty}\left|\mathbb{P}\left(\sup _{i q \in \bigcup_{l=1}^{n_{r}} I_{l}} X_{(r)}(i q) \leqslant u\right)-\mathbb{P}\left(\sup _{i q \in \bigcup_{l=1}^{n_{r}} I_{l}} Y_{(r)}(i q) \leqslant u\right)\right|=0 .
$$

Proof. Define $A=\mathbb{N} \cap \bigcup_{l=1}^{n_{r}} I_{l} q^{-1}=\left\{i_{1}, i_{2}, \ldots, i_{d}\right\}$, where $1 \leqslant i_{1}<i_{2}<$ $\ldots<i_{d}<\infty$, and observe that

$$
\begin{aligned}
\Delta_{(r)} & =\left|\mathbb{P}\left(\sup _{i q \in \bigcup_{l=1}^{n_{r}} I_{l}} X_{(r)}(i q) \leqslant u\right)-\mathbb{P}\left(\sup _{i q \in \bigcup_{l=1}^{n_{r}} I_{l}} Y_{(r)}(i q) \leqslant u\right)\right| \\
& =\left|\mathbb{P}\left(\sup _{i \in A} X_{(r)}(i q) \leqslant u\right)-\mathbb{P}\left(\sup _{i \in A} Y_{(r)}(i q) \leqslant u\right)\right| .
\end{aligned}
$$

For $i \in A$ and $1 \leqslant j \leqslant n$, we put $X_{i j}=X_{j}(i q)$ and $Y_{i j}=Y_{j}(i q)=X_{j}^{(\lfloor i q\rfloor+1)}(i q)$. Note that

$$
\begin{aligned}
\sigma_{i j, l k}^{X} & =\mathbb{E} X_{i j} X_{l k}=\mathbb{E} X_{j}(i q) X_{k}(l q)=r((i-l) q) \mathbb{I}\{j=k\}:=\sigma_{i l}^{X} \mathbb{I}\{j=k\}, \\
\sigma_{i j, l k}^{Y} & =\mathbb{E} Y_{i j} Y_{l k}=\mathbb{E} X_{j}^{(\lfloor i q\rfloor+1)}(i q) X_{k}^{(\lfloor l q\rfloor+1)}(l q) \\
& =r((i-l) q) \mathbb{I}\{\lfloor i q\rfloor=\lfloor l q\rfloor\} \mathbb{I}\{j=k\}:=\sigma_{i l}^{Y} \mathbb{I}\{j=k\} .
\end{aligned}
$$

It follows from Theorem 2.4 in [7] that

$$
\Delta_{(r)} \leqslant \frac{n\left(c_{n-1, r-1}\right)^{2}}{(2 \pi)^{n+1-r}} u^{-2(n-r)} \sum_{i, l \in A, i \neq l}\left|A_{i l}^{(r)}\right| \exp \left(-\frac{(n+1-r) u^{2}}{1+\rho_{i l}}\right),
$$

where

$$
\begin{gathered}
\rho_{i l}=\max \left\{\left|\sigma_{i l}^{X}\right|,\left|\sigma_{i l}^{Y}\right|\right\}=|r((i-l) q)|, \\
A_{i l}^{(r)}=\int_{\sigma_{i l}^{Y}}^{\sigma_{i l}^{X}} \frac{(1+|h|)^{2(n-r)}}{\left(1-h^{2}\right)^{(n+1-r) / 2}} d h \\
=\int_{0}^{r((i-l) q)} \frac{(1+|h|)^{2(n-r)}}{\left(1-h^{2}\right)^{(n+1-r) / 2}} d h \mathbb{I}\{\lfloor i q\rfloor \neq\lfloor l q\rfloor\} .
\end{gathered}
$$


Since $\delta:=\sup \{|r(t)|, t \geqslant \varepsilon\}<1$, for $i, l \in A$ satisfying $\lfloor i q\rfloor \neq\lfloor l q\rfloor$, one has $|(i-l) q| \geqslant \varepsilon$, and $|r((i-l) q)| \leqslant \delta<1$. Notice that the integrand in the definition of $A_{i l}^{(r)}$ is continuous and bounded on $[0, \delta]$, so there exists a constant $K_{1}$ such that

$$
\left|A_{i l}^{(r)}\right| \leqslant K_{1}|r((i-l) q)| \mathbb{I}\{\lfloor i q\rfloor \neq\lfloor l q\rfloor\}
$$

Hence,

$$
\begin{aligned}
\Delta_{(r)} \leqslant & \frac{n\left(c_{n-1, r-1}\right)^{2} K_{1}}{(2 \pi)^{n+1-r}} u^{-2(n-r)} \frac{\mathcal{T}_{r}}{q} \sum_{\varepsilon \leqslant k q \leqslant \mathcal{T}_{r}}|r(k q)| \exp \left(-\frac{(n+1-r) u^{2}}{1+|r(k q)|}\right) \\
= & \frac{n\left(c_{n-1, r-1}\right)^{2} K_{1}}{(2 \pi)^{n+1-r}} u^{-2(n-r)} \frac{\mathcal{T}_{r}}{q} \sum_{\varepsilon \leqslant k q \leqslant \mathcal{T}_{r}^{\beta}}|r(k q)| \exp \left(-\frac{(n+1-r) u^{2}}{1+|r(k q)|}\right) \\
& +\frac{n\left(c_{n-1, r-1}\right)^{2} K_{1}}{(2 \pi)^{n+1-r}} u^{-2(n-r)} \frac{\mathcal{T}_{r}}{q} \sum_{\mathcal{T}_{r}^{\beta}<k q \leqslant \mathcal{T}_{r}}|r(k q)| \exp \left(-\frac{(n+1-r) u^{2}}{1+|r(k q)|}\right) \\
= & : \mathbb{P}_{1}+\mathbb{P}_{2},
\end{aligned}
$$

where $0<\beta<(1-\delta) /(1+\delta)$.

First, we prove that $\mathbb{P}_{1} \rightarrow 0$ as $u \rightarrow \infty$. Indeed,

$$
\begin{aligned}
\mathbb{P}_{1} & \leqslant \frac{n\left(c_{n-1, r-1}\right)^{2} K_{1}}{(2 \pi)^{n+1-r}} u^{-2(n-r)} \frac{\mathcal{T}_{r}^{\beta+1}}{q^{2}} \exp \left(-\frac{(n+1-r) u^{2}}{1+\delta}\right) \\
& =\frac{n\left(c_{n-1, r-1}\right)^{2} K_{1}}{(2 \pi)^{n+1-r} a^{2}} u^{4 / \alpha-2(n-r)} \mathcal{T}_{r}^{\beta+1} \exp \left(-\frac{(n+1-r) u^{2}}{2}\right)^{2 /(1+\delta)} \\
& \leqslant K_{2} u^{4 / \alpha-2(n-r)+(\beta+1)(n+1-r-2 / \alpha)} \exp \left(\frac{(n+1-r) u^{2}}{2}\right)^{\beta-(1-\delta) /(1+\delta)} \\
& \rightarrow 0 \quad \text { as } u \rightarrow \infty .
\end{aligned}
$$

In order to show that $\mathbb{P}_{2} \rightarrow 0$, we put $\delta(t)=\sup \{|r(s) \log s|, s \geqslant t\}$. By (A3), we have $|r(t)| \leqslant \delta(t) / \log t$ and $\delta(t) \downarrow 0$ as $t \rightarrow \infty$. Moreover,

$$
\log \mathcal{T}_{r}=\frac{n+1-r}{2} u^{2}(1+o(1)) \quad \text { for } k q>\mathcal{T}_{r}^{\beta} .
$$

Thus,

$$
\begin{aligned}
\exp \left(-\frac{(n+1-r) u^{2}}{1+|r(k q)|}\right) & \leqslant \exp \left(-(n+1-r) u^{2}\left(1-\frac{\delta\left(\mathcal{T}_{r}^{\beta}\right)}{\log \mathcal{T}_{r}^{\beta}}\right)\right) \\
& \leqslant K_{3} \exp \left(-(n+1-r) u^{2}\right) .
\end{aligned}
$$


Hence,

$$
\begin{aligned}
\mathbb{P}_{2} \leqslant & \left\{K_{4} u^{-2(n-r)} \frac{\mathcal{T}_{r}^{2}}{q^{2}} \exp \left(-(n+1-r) u^{2}\right) \frac{1}{\log \mathcal{T}_{r}^{\beta}}\right\} \\
& \times \frac{q}{\mathcal{T}_{r}} \sum_{\mathcal{T}_{r}^{\beta}<k q \leqslant \mathcal{T}_{r}}|r(k q)| \log (k q) \\
\leqslant & K_{5} u^{-2(n-r)} \frac{u^{2(n+1-r-2 / \alpha)} \exp \left((n+1-r) u^{2}\right)}{u^{-4 / \alpha}} \exp \left(-(n+1-r) u^{2}\right) \frac{1}{u^{2}} \\
& \times \frac{q}{\mathcal{T}_{r}} \sum_{\mathcal{T}_{r}^{\beta}<k q \leqslant \mathcal{T}_{r}}|r(k q)| \log (k q) \\
\leqslant & K_{5} \frac{q}{\mathcal{T}_{r}} \sum_{\mathcal{T}_{r}^{\beta}<k q \leqslant \mathcal{T}_{r}}|r(k q)| \log (k q) \rightarrow 0 \quad \text { as } u \rightarrow \infty .
\end{aligned}
$$

This completes the proof.

\section{LEMMA 4.4. We have}

$$
\limsup _{u \rightarrow \infty}\left|\mathbb{P}\left(\sup _{i q \in \bigcup_{l=1}^{n_{r}} I_{l}} Y_{(r)}(i q) \leqslant u\right)-\mathbb{P}\left(\sup _{t \in\left[0, n_{r}\right]} Y_{(r)}(t) \leqslant u\right)\right| \leqslant x\left(\rho_{3}(a)+\varepsilon\right),
$$

where $\rho_{3}(a) \rightarrow 0$ as $a \rightarrow 0$.

Pr o of. Since $I_{l}, l=1,2, \ldots, n_{r}$, are disjoint, $\left\{Y_{(r)}(t), t \in I_{l}\right\}$ are independent, and, by stationarity,

$$
\begin{aligned}
0 & \leqslant \mathbb{P}\left(\sup _{i q \in \bigcup_{l=1}^{n_{r}} I_{l}} Y_{(r)}(i q) \leqslant u\right)-\mathbb{P}\left(\sup _{t \in \bigcup_{l=1}^{n_{r}} I_{l}} Y_{(r)}(t) \leqslant u\right) \\
& =\mathbb{P}\left(\sup _{i q \in[0,1-\varepsilon]} Y_{(r)}(i q) \leqslant u\right)^{n_{r}}-\mathbb{P}\left(\sup _{t \in[0,1-\varepsilon]} Y_{(r)}(t) \leqslant u\right)^{n_{r}} \\
& \leqslant n_{r}\left(\mathbb{P}\left(\sup _{i q \in I_{1}} Y_{(r)}(i q) \leqslant u\right)-\mathbb{P}\left(\sup _{t \in I_{1}} Y_{(r)}(t) \leqslant u\right)\right) \\
& \leqslant n_{r}\left(\mathbb{P}\left(Y_{(r)}(0)>u\right)+\mathbb{P}\left(\sup _{i q \in[0,1-\varepsilon]} Y_{(r)}(i q) \leqslant u\right)\right. \\
& \left.=x m_{r}(u)\left(o\left(\frac{1}{m_{r}(u)}\right)+\left(1-\frac{\mathcal{H}_{\alpha, n+1-r}^{\prime}(a)}{\mathcal{H}_{\alpha, n+1-r}}\right) \frac{1-\varepsilon}{\sup _{r}(u)} Y_{(r)}(t) \leqslant u\right)\right) \\
& \leqslant x\left(1-\frac{\mathcal{H}_{\alpha, n+1-r}^{\prime}(a)}{\mathcal{H}_{\alpha, n+1-r}}\right)=: x \rho_{3}(a),
\end{aligned}
$$


where $\rho_{3}(a) \rightarrow 0$ as $a \rightarrow 0$. Moreover,

$$
\begin{aligned}
0 & \leqslant \mathbb{P}\left(\sup _{t \in \bigcup_{l=1}^{n_{r}} I_{l}} Y_{(r)}(t) \leqslant u\right)-\mathbb{P}\left(\sup _{t \in\left[0, n_{r}\right]} Y_{(r)}(t) \leqslant u\right) \\
& \leqslant \mathbb{P}\left(\sup _{t \in[0,1-\varepsilon]} Y_{(r)}(t) \leqslant u\right)^{n_{r}}-\mathbb{P}\left(\sup _{t \in[0,1]} Y_{(r)}(t) \leqslant u\right)^{n_{r}} \\
& \leqslant n_{r} P\left(\sup _{t \in[0, \varepsilon]} Y_{(r)}(t)>u\right) \\
& =x m_{r}(u) \frac{\varepsilon}{m_{r}(u)}(1+o(1))=x \varepsilon(1+o(1)) .
\end{aligned}
$$

The combination of the above displays completes the proof.

LEMMA 4.5. We have

$$
\lim _{u \rightarrow \infty} \mathbb{P}\left(\sup _{t \in\left[0, n_{r}\right]} Y_{(r)}(t) \leqslant u\right)=e^{-x} .
$$

Proof. Since

$$
\begin{aligned}
\mathbb{P}\left(\sup _{t \in\left[0, n_{r}\right]} Y_{(r)}(t) \leqslant u\right) & =\mathbb{P}\left(\sup _{t \in[0,1]} X_{(r)}(t) \leqslant u\right)^{n_{r}} \\
& =\left(1-\mathbb{P}\left(\sup _{t \in[0,1]} X_{(r)}(t)>u\right)\right)^{n_{r}} \\
& =\left(1-m_{r}(u)^{-1}\right)^{x m_{r}(u)}(1+o(1)) \rightarrow e^{-x}
\end{aligned}
$$

the proof is completed.

Pro of of The or e $\mathrm{m}$ 3.1. The proof of the theorem follows directly from Lemmas 4.1-4.5.

LEMMA 4.6. For any $S>0$, we have

$$
\mathbb{P}\left(\sup _{t \in\left[0, S u^{-2 / \alpha}\right]} X_{(r)}(t)>u\right)=c_{n, r-1} \mathcal{H}_{\alpha, n+1-r}(S)(\Psi(u))^{n+1-r}(1+o(1))
$$

as $u \rightarrow \infty$.

The proof of Lemma 4.6 follows line-by-line the same reasoning as the proof of Theorem 2.2 in [8], and thus we omit it.

Proof of The ore m 3.2. (i) For any $t, u, S>0$, let us put $N_{t}=\left\lfloor\frac{t}{S u^{-2 / \alpha}}\right\rfloor \quad$ and $\quad \Delta_{k}=\left[k S u^{-2 / \alpha},(k+1) S u^{-2 / \alpha}\right]$ with $k=0,1, \ldots, N_{t}$. 
U p per bound. By stationarity of the process $\left\{X_{(r)}(t), t \geqslant 0\right\}$ and Lemma 1.6, we obtain

$$
\begin{aligned}
\mathbb{P}\left(\sup _{t \in[0, \mathcal{T}]} X_{(r)}(t)>u\right) & =\int_{0}^{\infty} \mathbb{P}\left(\sup _{s \in[0, t]} X_{(r)}(s)>u\right) d \mathbb{P}(\mathcal{T} \leqslant t) \\
& \leqslant \mathbb{P}\left(\sup _{s \in \Delta_{0}} X_{(r)}(s)>u\right)\left(\frac{u^{2 / \alpha}}{S} \int_{0}^{\infty} t d \mathbb{P}(\mathcal{T} \leqslant t)+1\right) \\
& =\frac{\mathcal{H}_{\alpha, n+1-r}(S)}{S} c_{n, r-1} \mathbb{E} \mathcal{T} u^{2 / \alpha}(\Psi(u))^{n+1-r}(1+o(1))
\end{aligned}
$$

as $u \rightarrow \infty$. Thus, letting $S \rightarrow \infty$, we get

$$
\mathbb{P}\left(\sup _{t \in[0, \mathcal{T}]} X_{(r)}(t)>u\right)=c_{n, r-1} s \mathcal{H}_{\alpha, n+1-r} u^{2 / \alpha} \mathbb{E} \mathcal{T}(\Psi(u))^{n+1-r}(1+o(1))
$$

L ow e r b o und. By Bonferroni's inequality, we have

$$
\begin{aligned}
& \mathbb{P}\left(\sup _{t \in[0, \mathcal{T}]} X_{(r)}(t)>u\right)=\int_{0}^{\infty} \mathbb{P}\left(\sup _{s \in[0, t]} X_{(r)}(s)>u\right) d \mathbb{P}(\mathcal{T} \leqslant t) \\
& \geqslant \int_{0}^{u} \mathbb{P}\left(\sup _{s \in[0, t]} X_{(r)}(s)>u\right) d \mathbb{P}(\mathcal{T} \leqslant t) \\
& \geqslant \mathbb{P}\left(\sup _{s \in \Delta_{0}} X_{(r)}(s)>u\right)\left(\frac{u^{2 / \alpha}}{S} \int_{0}^{u} t d \mathbb{P}(\mathcal{T} \leqslant t)-1\right) \\
& \quad-\int_{0}^{u} \sum_{0 \leqslant i<j \leqslant N_{t}} \mathbb{P}\left(\sup _{s \in \Delta_{i}} X_{(r)}(s)>u, \sup _{s \in \Delta_{j}} X_{(r)}(s)>u\right) d \mathbb{P}(\mathcal{T} \leqslant t) \\
& =: I_{1}-I_{2} .
\end{aligned}
$$

Note that

$$
I_{1}=\frac{\mathcal{H}_{\alpha, n+1-r}(S)}{S} c_{n, r-1} \mathbb{E} \mathcal{T} u^{2 / \alpha}(\Psi(u))^{n+1-r}(1+o(1))
$$

as $u \rightarrow \infty$. Thus, letting $S \rightarrow \infty$, we obtain

$$
I_{1} \geqslant c_{n, r-1} \mathcal{H}_{\alpha, n+1-r} u^{2 / \alpha} \mathbb{E} \mathcal{T}(\Psi(u))^{n+1-r} .
$$

Hence, in order to complete the proof it suffices to show that $I_{2}=o\left(I_{1}\right)$ as $u \rightarrow \infty$. 
Indeed, we have

$$
\begin{aligned}
I_{2}= & \int_{0}^{u} \sum_{k=1}^{N_{t}}\left(N_{t}-k\right) \mathbb{P}\left(\sup _{s \in \Delta_{0}} X_{(r)}(s)>u, \sup _{s \in \Delta_{k}} X_{(r)}(s)>u\right) d \mathbb{P}(\mathcal{T} \leqslant t) \\
\leqslant & \frac{u^{2 / \alpha}}{S} \int_{0}^{u} t d \mathbb{P}(\mathcal{T} \leqslant t) \sum_{k=1}^{N_{u}} \mathbb{P}\left(\sup _{s \in \Delta_{0}} X_{(r)}(s)>u, \sup _{s \in \Delta_{k}} X_{(r)}(s)>u\right) \\
\leqslant & \frac{u^{2 / \alpha}}{S} \mathbb{E} \mathcal{T} \sum_{k=1}^{N_{u}} \mathbb{P}\left(\sup _{s \in \Delta_{0}} X_{(r)}(s)>u, \sup _{s \in \Delta_{k}} X_{(r)}(s)>u\right) \\
\leqslant & c_{n, r-1} \frac{u^{2 / \alpha}}{S} \mathbb{E} \mathcal{T} \sum_{k=1}^{N_{u}} \mathbb{P}\left(\sup _{s \in \Delta_{0}} \min _{1 \leqslant i \leqslant n+1-r} X_{i}(s)>u, \sup _{s \in \Delta_{k}} X_{(r)}(s)>u\right) \\
\leqslant & c_{n, r-1} \frac{u^{2 / \alpha}}{S} \mathbb{E} \mathcal{T} \sum_{k=1}^{N_{u}} \mathbb{P}\left(\sup _{s \in \Delta_{0}} \min _{1 \leqslant i \leqslant n+1-r} X_{i}(s)>u, \sup _{s \in \Delta_{k}} \min _{1 \leqslant i \leqslant n+1-r} X_{i}(s)>u\right) \\
& +c_{n, r-1} \frac{u^{2 / \alpha}}{S} \mathbb{E} \mathcal{T} \sum_{k=1}^{N_{u}} \mathbb{P}\left(\sup _{s \in \Delta_{0}} \min _{1 \leqslant i \leqslant n+1-r} X_{i}(s)>u, \sup _{s \in \Delta_{k}} X_{(r)}(s)>u,\right. \\
= & \left.\sup _{s \in \Delta_{k}}+\min _{1 \leqslant i \leqslant n+1-r} X_{i}(s) \leqslant u\right)
\end{aligned}
$$

Since

$$
\begin{gathered}
\sum_{k=1}^{N_{u}} \mathbb{P}\left(\sup _{s \in \Delta_{0}} \min _{1 \leqslant i \leqslant n+1-r} X_{i}(s)>u, \sup _{s \in \Delta_{k}} \min _{1 \leqslant i \leqslant n+1-r} X_{i}(s) \leqslant u, \sup _{s \in \Delta_{k}} X_{(r)}(s)>u\right) \\
\leqslant N_{u} \mathbb{P}\left(\sup _{s \in \Delta_{0}} X_{1}(s)>u\right)^{n+2-r}
\end{gathered}
$$

we get $I_{22}=o\left(I_{1}\right)$ as $u \rightarrow \infty$. Moreover, using the relations

$$
\begin{gathered}
I_{21} \leqslant c_{n, r-1} \frac{u^{2 / \alpha}}{S} \mathbb{E} \mathcal{T} \sum_{k=1}^{N_{u}} \mathbb{P}\left(\sup _{s \in \Delta_{0}} X_{1}(s)>u, \sup _{s \in \Delta_{k}} X_{1}(s)>u\right)^{n+r-1} \\
\leqslant c_{n, r-1} u^{2 / \alpha} \mathbb{E} \mathcal{T}\left(\frac{1}{S^{1 /(n+r-1)}} \sum_{k=1}^{N_{u}} \mathbb{P}\left(\sup _{s \in \Delta_{0}} X_{1}(s)>u, \sup _{s \in \Delta_{k}} X_{1}(s)>u\right)\right)^{n+r-1}
\end{gathered}
$$

we are left with finding a tight asymptotic bound for

$$
\frac{1}{S^{1 /(n+r-1)}} \sum_{k=1}^{N_{u}} \mathbb{P}\left(\sup _{s \in \Delta_{0}} X_{1}(s)>u, \sup _{s \in \Delta_{k}} X_{1}(s)>u\right),
$$

which follows by the same argument as that given in the proof of Theorem D.2 in [12] (see also the proof of Theorem 3.1 in [4]), with the minor exception that the 
first term in the above summand is bounded by

$$
\begin{aligned}
\mathbb{P}\left(\sup _{s \in \Delta_{0}} X_{1}(s)>u, \sup _{s \in \Delta_{1}} X_{1}(s)>u\right) & \\
\leqslant & \mathbb{P}\left(\sup _{s \in\left[0, S u^{-2 / \alpha}\right]} X_{1}(s)>u, \sup _{\left[\left(S+S^{1 /(2(n+r-1))}\right) u^{-2 / \alpha},\right.} X_{1}(s)>u\right) \\
& +\mathbb{P}\left(\sup _{s \in\left[0, S^{1 /(2(n+r-1))} u^{-2 / \alpha}\right]} X_{1}(s)>u\right) .
\end{aligned}
$$

This completes the proof of Theorem 3.1(i).

(ii) For any $0<A<B<\infty$ and sufficiently large $u$, we make the following decomposition:

$$
\begin{aligned}
\mathbb{P}\left(\sup _{t \in[0, \mathcal{T}]} X_{(r)}(t)>u\right) & \\
= & \left(\int_{0}^{A m_{r}(u)}+\int_{A m_{r}(u)}^{B m_{r}(u)}+\int_{B m_{r}(u)}^{\infty}\right) \mathbb{P}\left(\sup _{s \in[0, t]} X_{(r)}(s)>u\right) d \mathbb{P}(\mathcal{T} \leqslant t) \\
= & : I_{1}+I_{2}+I_{3} .
\end{aligned}
$$

We analyze $I_{1}, I_{2}, I_{3}$ separately.

Integral $I_{1}$. Since the process $\left\{X_{(r)}(t), t \geqslant 0\right\}$ is stationary, by Bonferroni's inequality, we have

$$
\begin{aligned}
I_{1} \leqslant & \mathbb{P}\left(\sup _{s \in[0,1]} X_{(r)}(s)>u\right)\left(\int_{0}^{A m_{r}(u)} t d \mathbb{P}(\mathcal{T} \leqslant t)+1\right) \\
= & \mathbb{P}\left(\sup _{s \in[0,1]} X_{(r)}(s)>u\right) \\
& \times\left(\int_{0}^{A m_{r}(u)} \mathbb{P}(\mathcal{T}>t) d t-A m_{r}(u) \mathbb{P}\left(\mathcal{T}>A m_{r}(u)\right)+1\right) .
\end{aligned}
$$

Using Karamata's theorem, we get

$$
\int_{0}^{A m_{r}(u)} \mathbb{P}(\mathcal{T}>t) d t=\frac{1}{\lambda} A m_{r}(u) \mathbb{P}\left(\mathcal{T}>A m_{r}(u)\right)(1+o(1)) \quad \text { as } u \rightarrow \infty,
$$

which, combined with (4.6) and Theorem 2.2 in [8], implies that

$$
\begin{aligned}
I_{1} & \leqslant \frac{\lambda}{1-\lambda} A \mathbb{P}\left(\mathcal{T}>A m_{r}(u)\right)(1+o(1)) \\
& =\frac{\lambda}{1-\lambda} A^{1-\lambda} \mathbb{P}\left(\mathcal{T}>m_{r}(u)\right)(1+o(1)) \quad \text { as } u \rightarrow \infty .
\end{aligned}
$$


Integral $I_{3}$. It is straightforward that

$$
I_{3} \leqslant \mathbb{P}\left(\mathcal{T}>B m_{r}(u)\right)(1+o(1))=B^{-\lambda} \mathbb{P}\left(\mathcal{T}>m_{r}(u)\right)(1+o(1)) \quad \text { as } u \rightarrow \infty .
$$

In te g r a l $I_{2}$. For any $\varepsilon>0$ and sufficiently large $u$, applying Theorem B.], we get the upper bound

$$
\begin{aligned}
I_{2}= & \int_{A}^{B} \mathbb{P}\left(\sup _{s \in\left[0, x m_{r}(u)\right]} X_{(r)}(s)>u\right) d \mathbb{P}\left(\mathcal{T} \leqslant x m_{r}(u)\right) \\
\leqslant & (1+\varepsilon) \int_{A}^{B}\left(1-e^{-x}\right) d \mathbb{P}\left(\mathcal{T} \leqslant x m_{r}(u)\right) \\
= & (1+\varepsilon) \int_{A}^{B} e^{-x} \mathbb{P}\left(\mathcal{T}>x m_{r}(u)\right) d x-(1+\varepsilon)\left(1-e^{-B}\right) \mathbb{P}\left(\mathcal{T}>B m_{r}(u)\right) \\
& +(1+\varepsilon)\left(1-e^{-A}\right) \mathbb{P}\left(\mathcal{T}>A m_{r}(u)\right)
\end{aligned}
$$

and similarly we obtain the lower bound

$$
\begin{aligned}
I_{2} \geqslant & (1-\varepsilon) \int_{A}^{B} e^{-x} \mathbb{P}\left(\mathcal{T}>x m_{r}(u)\right) d x-(1-\varepsilon)\left(1-e^{-B}\right) \mathbb{P}\left(\mathcal{T}>B m_{r}(u)\right) \\
& +(1-\varepsilon)\left(1-e^{-A}\right) \mathbb{P}\left(\mathcal{T}>A m_{r}(u)\right) .
\end{aligned}
$$

Since $\mathcal{T}$ has a regularly varying tail distribution at infinity, by Theorem 1.5.2 in [5], we get

$$
\int_{A}^{B} e^{-x} \mathbb{P}\left(\mathcal{T}>x m_{r}(u)\right) d x=\mathbb{P}\left(\mathcal{T}>m_{r}(u)\right) \int_{A}^{B} e^{-x} x^{-\lambda} d x(1+o(1)) \quad \text { as } u \rightarrow \infty .
$$

Thus, for any $\varepsilon>0$ and $0<A<B<\infty$, we obtain

$$
\begin{aligned}
\limsup _{u \rightarrow \infty} \frac{I_{2}}{\mathbb{P}\left(\mathcal{T}>m_{r}(u)\right)} & \\
& \leqslant(1+\varepsilon)\left(\int_{0}^{B} x^{-\lambda} e^{-x} d x-\left(1-e^{-B}\right) B^{-\lambda}+\left(1-e^{-A}\right) A^{-\lambda}\right)
\end{aligned}
$$

and

$$
\begin{aligned}
\liminf _{u \rightarrow \infty} \frac{I_{2}}{\mathbb{P}\left(\mathcal{T}>m_{r}(u)\right)} \\
\quad \leqslant(1-\varepsilon)\left(\int_{0}^{B} x^{-\lambda} e^{-x} d x-\left(1-e^{-B}\right) B^{-\lambda}+\left(1-e^{-A}\right) A^{-\lambda}\right) .
\end{aligned}
$$


Therefore, letting $A \rightarrow 0, B \rightarrow \infty$, and $\varepsilon \rightarrow 0$, we find that $I_{1}$ and $I_{3}$ are negligible, and

$$
I_{2}=\Gamma(1-\lambda) \mathbb{P}\left(\mathcal{T}>m_{r}(u)\right)(1+o(1)) \quad \text { as } u \rightarrow \infty,
$$

which completes the proof of Theorem 3.2(ii).

(iii) L o w e r b o u nd. From Theorem B.], for any given $B>0$, it follows that

$$
\begin{aligned}
\mathbb{P}\left(\sup _{t \in[0, \mathcal{T}]} X_{(r)}(t)>u\right) & \geqslant \mathbb{P}\left(\sup _{s \in\left[0, B m_{r}(u)\right]} X_{(r)}(s)>u\right) \mathbb{P}\left(\mathcal{T}>B m_{r}(u)\right) \\
& =\left(1-e^{-B}\right) \mathbb{P}\left(\mathcal{T}>m_{r}(u)\right)(1+o(1))
\end{aligned}
$$

as $u \rightarrow \infty$. Thus, letting $B \rightarrow \infty$, we obtain the asymptotic lower bound

$$
\mathbb{P}\left(\sup _{t \in[0, \mathcal{T}]} X_{(r)}(t)>u\right) \geqslant \mathbb{P}\left(\mathcal{T}>m_{r}(u)\right)(1+o(1)) \quad \text { as } u \rightarrow \infty .
$$

U p per b o und. For given $A>0$, we get

$$
\begin{aligned}
& \mathbb{P}\left(\sup _{t \in[0, \mathcal{T}]} X_{(r)}(t)>u\right) \\
& \leqslant \int_{0}^{A m_{r}(u)} \mathbb{P}\left(\sup _{s \in[0, t]} X_{(r)}(s)>u\right) d \mathbb{P}(\mathcal{T} \leqslant t)+\mathbb{P}\left(\mathcal{T}>A m_{r}(u)\right) \\
& =\int_{0}^{A m_{r}(u)} \mathbb{P}\left(\sup _{s \in[0, t]} X_{(r)}(s)>u\right) d \mathbb{P}(\mathcal{T} \leqslant t)+\mathbb{P}\left(\mathcal{T}>m_{r}(u)\right)(1+o(1))
\end{aligned}
$$

as $u \rightarrow \infty$. Due to the stationarity of the process $\left\{X_{(r)}(t), t \geqslant 0\right\}$ and Bonferroni's inequality, we have

$$
\begin{aligned}
\int_{0}^{A m_{r}(u)} \mathbb{P}\left(\sup _{s \in[0, t]} X_{(r)}(s)>u\right) d \mathbb{P}(\mathcal{T} \leqslant t) \\
\quad \leqslant \mathbb{P}\left(\sup _{s \in[0,1]} X_{(r)}(s)>u\right)\left(\int_{0}^{A m_{r}(u)} t d \mathbb{P}(\mathcal{T} \leqslant t)+1\right) \\
\quad \leqslant \mathbb{P}\left(\sup _{s \in[0,1]} X_{(r)}(s)>u\right)\left(\int_{0}^{A m_{r}(u)} \mathbb{P}(\mathcal{T}>t) d t+1\right) .
\end{aligned}
$$

From Karamata's theorem (see, e.g., Proposition 1.5.8 in [5]), we get

$$
\int_{0}^{A m_{r}(u)} \mathbb{P}(\mathcal{T}>t) d t=A m_{r}(u) \mathbb{P}\left(\mathcal{T}>A m_{r}(u)\right)(1+o(1))
$$


as $u \rightarrow \infty$, which, combined with (4.7) and Theorem 2.2 in [8], implies that

$$
\mathbb{P}\left(\sup _{t \in[0, \mathcal{T}]} X_{(r)}(t)>u\right) \leqslant(1+A) \mathbb{P}\left(\mathcal{T}>m_{r}(u)\right)(1+o(1))
$$

as $u \rightarrow \infty$. Letting $A \rightarrow 0$, we obtain (B.4). This completes the proof of Theorem 3.2.

Acknowledgments. The author would like to thank Professor Krzysztof Dębicki and the referees for their valuable comments.

\section{REFERENCES}

[1] J. M. P. Albin and H. Choi, A new proof of an old result by Pickands, Electron. Commun. Probab. 15 (2010), pp. 339-345.

[2] M. T. Alodat, An approximation to cluster size distribution of two Gaussian random fields conjunction with application to FMRI data, J. Statist. Plann. Inference 141 (2011), pp. 23312347.

[3] M. T. Alodat, M. Al-Rawwash, and M. A. Jebrini, Duration distribution of the conjunction of two independent F processes, J. Appl. Probab. 47 (2010), pp. 179-190.

[4] M. Arendarczyk and K. Dębicki, Exact asymptotics of supremum of a stationary Gaussian process over a random interval, Statist. Probab. Lett. 82 (2012), pp. 645-652.

[5] N. H. Bingham, C. M. Goldie, and J. L. Teugels, Regular Variation, Cambridge University Press, Cambridge 1989.

[6] D. Cheng and Y. Xiao, Excursion probability of Gaussian random fields on sphere, Bernoulli 22 (2016), pp. 1113-1130.

[7] K. Dębicki, E. Hashorva, L. Ji, and C. Ling, On Berman's inequality for order statistics of Gaussian arrays, submitted.

[8] K. Dębicki, E. Hashorva, L. Ji, and K. Tabiś, On the probability of conjunctions of stationary Gaussian processes, Statist. Probab. Lett. 88 (2014), pp. 141-148.

[9] K. Dębicki, E. Hashorva, L. Ji, and K. Tabiś, Extremes of vector-valued Gaussian processes: Exact asymptotics, Stochastic Process. Appl. 125 (2015), pp. 4039-4065.

[10] J. Galambos, Bonferroni inequalities, Ann. Probab. (1977), pp. 577-581.

[11] M. R. Leadbetter, G. Lindgren, and H. Rootzén, Extremes and Related Properties of Random Sequences and Processes, Springer, New York 1983.

[12] V. I. Piterbarg, Asymptotic Methods in the Theory of Gaussian Processes and Fields, Transl. Math. Monogr., Vol. 148, American Mathematical Society, Providence 1996.

[13] Z. Tan and E. Hashorva, Limit theorems for extremes of strongly dependent cyclostationary $\chi$-processes, Extremes 16 (2) (2013), pp. 241-254.

[14] K. J. Worsley and K. J. Friston, A test for a conjunction, Statist. Probab. Lett. 47 (2000), pp. $135-140$.

Chunming Zhao

Department of Statistics, School of Mathematics

Southwest Jiaotong University

Xi' an Road 999, Xipu, Pixian

Chengdu, Sichuan 611756, PR of China

E-mail: cmzhao@swjtu.cn 